\title{
Magnesium: An Essential Nutrient for a Good Biomaterial
}

\author{
Michele V. Manuel and Norbert Hort
}

Fueled by the needs of an aging population, biomedical applications of magnesium and its alloys have been steadily increasing every year. Numbers from the U.S. Census Bureau indicate that by 2030,1 in 5 people living in the U.S. will be 65 and older. ${ }^{1}$ This statistic is contrasted by the fact that $80 \%$ of people within this age group suffer from at least one chronic condition, thus creating a driving force for the two largest medical sectors: orthopedics and cardiovascular devices. ${ }^{2}$ As the median age for life expectancy increases, patients are beginning to outlive their implanted medical devices. This places a unique demand on medical researchers to develop new implant technologies that will either outlive the patient, have the capability of being removed at a later date, or reabsorb into the body when they are no longer needed. Magnesium as a biomaterial fits within the last category and is of great interest for its potential use in orthopedic and cardiovascular applications.

Being one of the most abundant ions in the human body, magnesium has attracted much attention due to its therapeutic properties. Magnesium is critical for many cell-mediated processes and its deficiency can led to many chronic diseases. When compared to its ceramic and polymeric counterparts, magnesium boasts a modulus and strength similar to bone which reduces the risk of stress shielding in orthopedic applications while lowering the total amount of foreign material needed to create high performance cardiovascular devices. ${ }^{3,4}$ The limitations of this technology include a rapid dissolution rate that is shorter than the healing rate of the surrounding tissue (typically complete absorption of a magnesium cardiovascular stent occurs within 2-4 months ${ }^{5}$ ) and the use of common alloying elements with disputed biocompatibility. These elements include aluminum and rare earth species, that are used to enhance the properties of magnesium.

To shed light on this growing field, the TMS Magnesium Committee organized a special symposium at the TMS 2011 Annual Meeting \& Exhibition in San Diego on the biomedical applications of magnesium alloys. Researchers from a number of leading international institutions provided talks on the stateof-the-art of the science in this field and to exchange novel scientific ideas. These institutions included national/ international laboratories, universities, and industry. The proceeding papers in this special issue highlight the research perspective of two of these groups.

The paper by Harpreet S. Brar et al. discusses the use of a systems design approach to develop new orthopedic magnesium devices. The article provides a holistic view of the complexity and intricacies involved in the development of hierarchically structured materials for dynamic environments such as the human body.
To address this issue, the authors provide a biomedical perspective and translation of the materials design approach that has been successfully demonstrated in the aerospace and defense industries.

The paper by Yufeng Zheng and Xuenan Gu provides a timely review of the current developments in magnesium biomedical research in China. China, being one of the fastest growth areas for magnesium research, has dramatically increased its biomedical magnesium research activity over the past several years. The article covers novel alloy systems, geometric structures, and surface modification approaches. It provides a great starting point and succinct review of the field.

\section{References}

1. U.S. Census Bureau American Community Survey: 2008 Selected Characteristics in the United States (Washington, D.C.: U.S. Census Bureau, 2008).

2. M. Much, Investor's Business Daily (2008) p. A09.

3. B. Heublein et al., Heart, 89 (2003), pp. 651-656.

4. J.R. Davis, editor, Handbook of Materials for Medical Devices (Materials Park, OH: ASM International, 2003).

5. H. Hamid and J. Coltart, McGill Journal of Medicine, 10 (2) (2007), pp. 105-111.

6. P. Peeters et al., J. Endovascular Specialists, 12 (2005), pp. 1-5.

Michele V. Manuel is with the Department of Materials Science and Engineering, University of Florida, Gainesville, Florida, 32611; Norbert Hort is the head of the department in the magnesium processing group, MagIC-Magnesium Innovation Center, Institute of Materials Research, Geesthacht, Germany. They are the advisors to JOM from the Magnesium Committee of the Light Metals Division of TMS.

Michele V. Manuel and Norbert Hort are TMS Members!

To read more about them, turn to page 7. To join TMS, visit www.tms.org/Society/Membership.aspx. 